\title{
Thermodynamics of ligand binding to proteins
}

\author{
Stanley J. Gill \\ Department of Chemistry and Biochemistry, University of Colorado, Boulder CO. \\ 80309-0215, USA
}

\begin{abstract}
The phenomenological characterization of binding reactions is described by the amount of chemical ligand bound along with concomitant and physical effects of heat and volume change. Binding measurements, calorimetry, and dilatometry provide a direct route for the evaluation of relevant reaction parameters. Indirectly the study of the response to temperature, pressure, and ligand chemical potentials yields the same information. From a theoretical view the functional chemistry of biological molecules is broadly depicted by a stoichiometrically based binding partition function as suggested by Wyman (Adv. Protein Chem., 19. 224-286 (1964)). Derivatives of this function provide a direct link to experimental measurements and provide the basis for the quantitative description of homotropic cooperativity and heterotropic linkage. The formulation of the partition function can when appropriate be given for allosteric (conformational) and polysteric (aggregational) systems, with the inclusion of binding effects involving chemical, volume, and enthalpy change. Applications of these concepts are drawn from recent studies of ligand binding to hemoglobin and hemocyanin.
\end{abstract}

\section{INTRODUCTION}

Biological macromolecules function in a variety of modes. An essential facet is the involvement of binding reactions by regulatory, usually simple, ligands. Substrate binding to enzymes and oxygen binding to respiratory proteins are examples. Underlying these processes one often finds coupling of auxiliary reactions to the one of primary concern. Such interaction leads to the phenomena of linkage between different (heterotropic) ligands and is of fundamental importance in enabling biological macromolecules to execute control and regulation of complex systems. Equally significant is the property of cooperativity found in certain multisubunit biological molecules where the same ligand facilitates binding of another.of the same kind. This is known as homotropic effect. Underlying often deceptively simple ligand binding reactions, one sometimes finds the macromolecule changing its conformational and aggregational state. The exploration and characterization of these events is greatly facilitated by the methods of classical and statistical thermodynamics.

In this lecture I wish to outline the description of multiple equilibrium events, that describe typical biological processes, in terms of a general binding partition function (ref. 1-4). This function, which may incorporate known features of structure and reaction properties of a given system, has a direct connection to measurable thermodynamic properties, such as the amount of ligand binding and the enthalpy change of ligand binding. The application of suitable binding partition functions to results obtained for oxygen and carbon monoxide binding to hemoglobin and hemocyanins allows description of the binding potential $\pi$ (ref. 5) in terms of the chemical potentials of these ligands. From the binding potential one obtains by differentiation the full description of binding, linkage, and cooperativity that a given macromolecular system exhibits. 


\section{THE MACROMOLECULAR BINDING PARTITION FUNCTION}

The macromolecular binding partition function, designated by the symbol $Q$, represents the sum of the molar concentrations of all macromolecular species in the system (ref. 6). A macromolecular species will be represented by the symbol $\mathrm{M}_{\mathrm{k}, \mathrm{h}} \mathrm{X}_{\mathrm{i}} \mathrm{Y}_{\mathrm{j}}$, and its molar concentration by $\left[\mathrm{M}_{\mathrm{k}, \mathrm{h}} \mathrm{X}_{\mathrm{i}} \mathrm{Y}_{\mathrm{j}}\right]$. This notation designates a macromolecular species with $\mathrm{i}$ ligands of type $X$ bound and $j$ ligands of type $Y$ bound. The use of additional subscripts $k$ and $h$ will describe macromolecular species with different allosteric forms ( $k$ ), and to designate different degrees of subunit association (h). The partition function is represented as follows:

$$
\mathrm{Q}=\Sigma\left[\mathrm{M}_{\mathrm{k}, \mathrm{h}} \mathrm{X}_{\mathrm{i}} \mathrm{Y}_{\mathrm{j}}\right]
$$

where the summation is taken over all relevant subscripts, depending upon the nature of the problem. The macromolecular species concentration is in general a function of ligand activities ( $x$ and $y$ ), subunit concentration $(m)$, temperature $(T)$ and pressure $(p)$. For a non-dissociating macromolecular system with $r$ subunits, the total subunit concentration $\mathrm{m}$ is simply equal to $\mathrm{r} \mathrm{Q}$.

\section{Single ligand; non-dissociating macromolecule}

For a non-dissociating macromolecule with $t$ binding sites for ligand $X$, one may describe the binding reactions by successive additions of $X$ to the unligated macromolecule $\mathrm{M}$ as follows:

$$
\mathrm{M} \rightarrow \mathrm{MX} \rightarrow \mathrm{MX}_{2} \rightarrow \ldots \rightarrow \mathrm{MX}_{\mathrm{t}}
$$

The reaction process of this set of species, starting from $M$, is $M+i X \rightarrow M X_{i}$, and the equilibrium constant for this overall reaction will be denoted by $\beta_{\mathrm{i}}$. Alternatively, one may describe the reaction processes in terms of successive stepwise reactions, $\mathrm{M}_{\mathrm{i}-1}+\mathrm{X}$ $\rightarrow \mathrm{MX}_{\mathrm{i}}$, with an equilibrium constant $\mathrm{K}_{\mathrm{i}}$. The two descriptions are complementary with $K_{i}=\beta_{i} / \beta_{i-1}$ or with $\beta_{i}=K_{1} K_{2} \ldots K_{i}$. The amount of $X$ ligand bound per mole of reaction sites, denoted by $X_{m}$, is found by the sum of the concentrations of the various ligated species, weighted according to the number of ligands carried, and divided by total concentration of reaction sites. The species concentrations are given by $\left[M X_{i}\right]=[M] \beta_{i} x^{i}$; thus amount of $\mathrm{X}$ ligand bound per reaction site is

$$
\mathrm{X}_{\mathrm{m}}=\frac{\sum \mathrm{i}\left[\mathrm{MX}_{\mathrm{i}}\right]}{\mathrm{t} \sum\left[\mathrm{MX}_{\mathrm{i}}\right]}=\frac{\sum \mathrm{i} \beta_{\mathrm{i}} \mathrm{x}^{\mathrm{i}}}{\mathrm{t} \sum \beta_{\mathrm{i}} \mathrm{x}^{\mathrm{i}}}=\frac{\mathrm{x}}{\mathrm{t} \sum\left[\mathrm{MX}_{\mathrm{i}}\right]} \frac{\partial \sum\left[\mathrm{MX} \mathrm{i}_{\mathrm{i}}\right]}{\partial \mathrm{x}}=\frac{1}{\mathrm{t}} \frac{\partial \ln \mathrm{Q}}{\partial \ln \mathrm{x}}
$$

where the final partial derivative form is found using the definition of $Q$ by equation (1). For this situation we see that $Q=[M] \Sigma \beta_{i} x^{i}$. The fraction of the ith species $\alpha_{i}$ is given by the ratio $\beta_{i} x^{i} / \Sigma \beta_{i} x^{i}$.

A plot of $\mathrm{X}_{\mathrm{m}}$ versus $\log \mathrm{x}$ is shown for oxygen binding to hemoglobin in Fig. 1. Also shown is the derivative of the binding curve. The derivative is related to the reaction parameters and the partition function as follows:

$$
\frac{d x_{m}}{d \ln x}=\frac{\sum i^{2} \beta_{i} x^{i} \sum \beta_{i} x^{i}-\left(\sum i \beta_{i} x^{i}\right)^{2}}{t\left(\sum \beta_{i} x^{i}\right)^{2}}=\frac{1}{t} \frac{\partial^{2} \ln Q}{\partial(\ln x)^{2}}
$$

The derivative $\mathrm{dX}_{\mathrm{m}} / \mathrm{dlnx}$ multiplied by the number of binding sites $\mathrm{t}$ is known as the binding capacity of the macromolecule (ref. 9) and describes the cooperativity of the macromolecule. It is closely related to the slope of the Hill plot, $\mathrm{n}_{\mathrm{H}}$, which is a common expression of cooperativity (ref. 10):

$$
\mathrm{n}_{\mathrm{H}}=\frac{1}{\mathrm{X}_{\mathrm{m}}\left(1-\mathrm{X}_{\mathrm{m}}\right)} \frac{\mathrm{d} \mathrm{X}_{\mathrm{m}}}{\mathrm{d} \ln \mathrm{x}}
$$



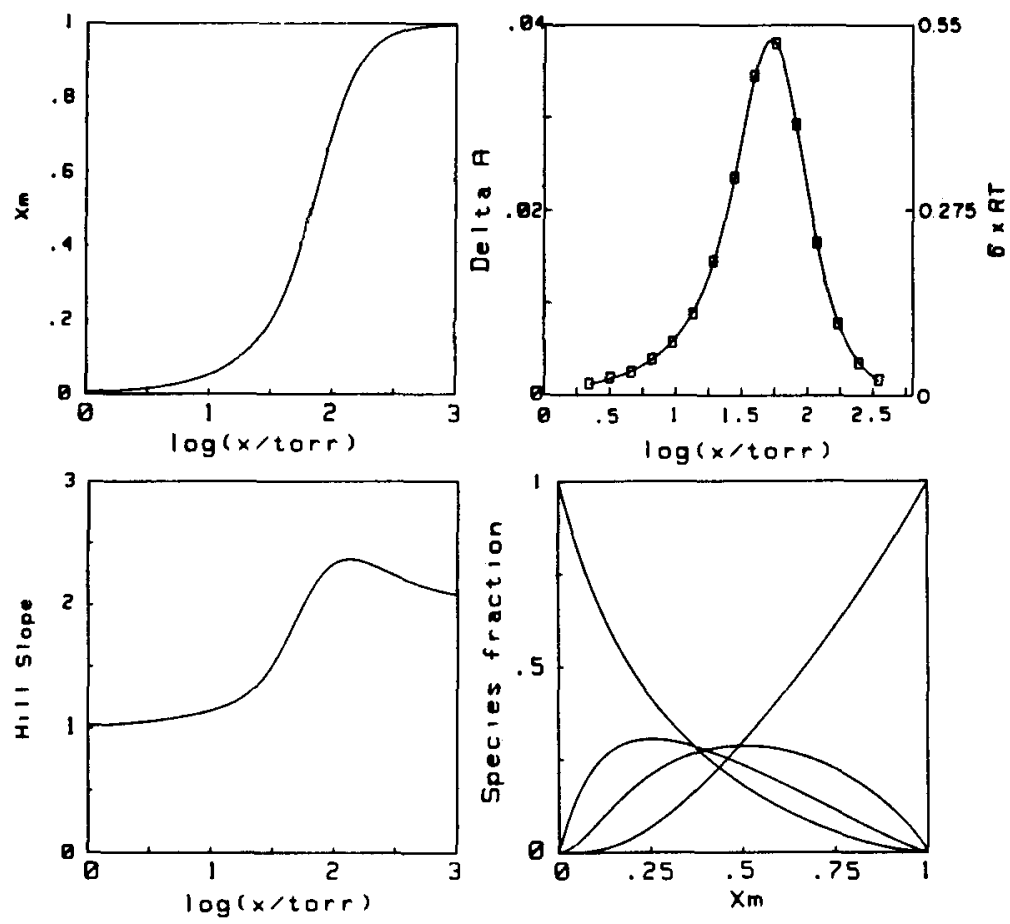

Fig. 1 Oxygen binding to human hemoglobin. (Top left) Moles of oxygen bound per mole of binding site, $X_{m}$, as a function of the logarithm of oxygen partial pressure. (Top right) Derivative binding curve as a function of the logarithm of oxygen partial pressure. Absorbance change data ( $\square$ ) fitted to solid line with $\beta_{1}=$ 0.019 Torr $^{-1}, \beta_{2}=0.00034$ Torr $^{-2}, \beta_{3}=0$ Torr $^{-3}, \beta_{4}=7.57 \times 10^{-7}$ Torr $^{-4}$, and overall optical absorbance change for complete reaction is 0.1972 . (Lower left) Hill coefficient $n_{H}$ computed as a function of logarithm of oxygen partial pressure. (Lower right) Species fraction plot as a function of moles oxygen bound per mole of hemoglobin., $t X_{m}$. Experimental conditions: $2 \mathrm{mM}$ heme, 0.1 Hepes, $1 \mathrm{mM}$ Na 2 EDTA,0.1 M NaCl, 10mM IHP, pH 7.0, 250 C. (ref. 7, 8).

A value of $n_{H}$ greater than one indicates positive cooperativity or interactions between binding sites. The results shown in Fig. 1 were obtained by use of a thin layer optical cell system incorporating a high precision dilution valve for precise change of gas partial pressure (ref. 11). The optical cell and valve along with a calibration plot showing the logarithmic change of partial pressure with execution of step dilutions are depicted in Fig. 2.

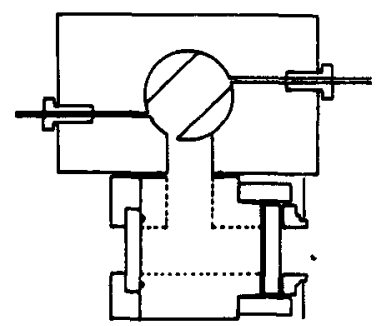

Cell Flush Position
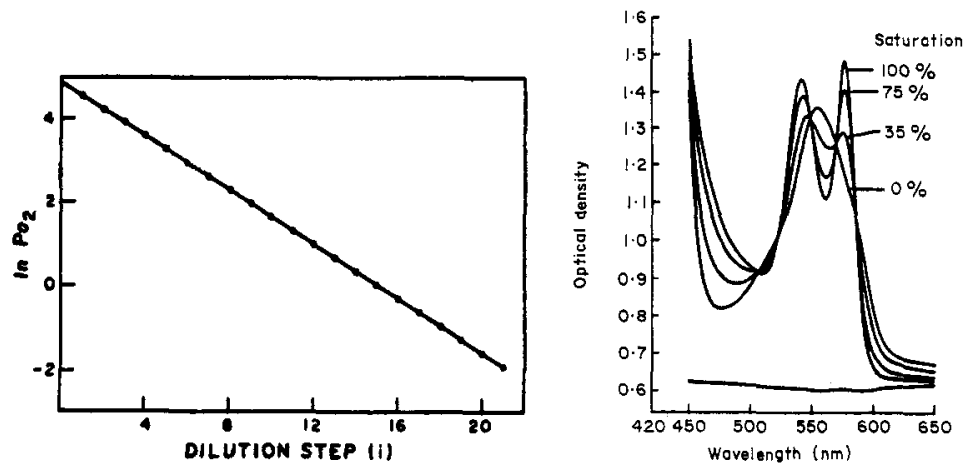

Fig 2. Thin layer sample optical cell and dilution valve system. (Left) Schematic diagram of apparatus (ref. 11). (Middle) Logarithmic plot of oxygen partial pressure (measured by an oxygen electrode) as a function of stepwise dilution using nitrogen. The best fit theoretical line is determined from the predicted partial pressure $p_{i}$ at the end of diluting the system by $i$ steps, starting at a pressure of $p_{0}$, each step involving a dilution factor $\mathrm{D}: \mathrm{p}_{i}=\mathrm{p}_{\mathrm{o}} \mathrm{D}^{\mathrm{i}}$. (Right) Optical absorbance spectra of hemoglobin at different oxygen saturations. 


\section{Generalized binding properties}

In a more general sense the binding properties of the macromolecule are described by the amount of a given ligand bound or by the enthalpy or volume the system has. One may compute these operational properties from appropriate derivatives of the partition function (ref. 12, 13). For convenience we shall define a subunit as consisting a single $X$ binding site. As we have just seen the amount of ligand $X$ bound per mole of subunit, $e$. g. $X_{m}$, may be calculated from the dependence of $Q$ upon the ligand activity $x$. The enthalpy per mole of subunit, e. $g . H_{m}$, is obtained from the dependence of $Q$ upon the reciprocal temperature $1 / T$, and the volume per mole of subunit, e. $g . V_{m}$, is given by the dependence upon the pressure. All of these quantities are evaluated with reference to a chosen standard state, denoted by a superscript zero.

In the situation where the macromolecule can either dissociate or associate the sum of macromolecular species concentration will depend upon the amount of solvent and other ligand activities. However for a given solution the total subunit concentration will be fixed and will be determined by the subunit concentration $m$. Thus we shall determine the binding parameters of the system in terms of subunits. With these considerations the explicit form of the equations is summarized by the second column as follows:

$$
\begin{aligned}
& X_{m}-X_{m}^{0}=\frac{x}{m} \frac{\partial Q}{\partial x}=-\frac{\partial \mu_{M}}{\partial \mu_{x}} \\
& H_{m}-H_{m}^{o}=-\frac{R}{m} \frac{\partial Q}{\partial 1 / T}=\frac{\partial\left(\mu_{M} / T\right)}{\partial(1 / T)} \\
& V_{m}-V_{m}^{o}=-\frac{R T}{m} \frac{\partial Q}{\partial p}=\frac{\partial \mu_{M}}{\partial p}
\end{aligned}
$$

The second form of these equations arise from a relation between the chemical potential of the macromolecule $\mu_{M}$ (per mole of subunit) and the binding partition function of the macromolecule, namely $d \mu_{M}=-(R T / m) d Q$ and that the derivative relation between the chemical potential $\left(\mu_{x}\right)$ and the ligand activity $x$ is $d \mu_{x}=R T d \ln x$. When the macromolecule is non-dissociating then $m=t Q$ and $d \mu_{M}=-(R T / t) d \ln Q$.

The thermodynamic origin of these equations comes from an expression of the GibbsDuhem equation in terms of reciprocal temperatures (ref. 14):

$$
0=n_{M} d\left(\frac{\mu_{M}}{T}\right)+n_{x} d\left(\frac{\mu_{x}}{T}\right)-H d\left(\frac{1}{T}\right)-\frac{V}{T} d p
$$

Upon normalization by the moles of subunits, $n_{M}$, one obtains

$$
-d\left(\frac{\mu_{M}}{T}\right)=X_{m} d\left(\frac{\mu_{x}}{T}\right)-H_{m} d\left(\frac{1}{T}\right)-\frac{V_{m}}{T} d p
$$

The partial derivative expressions of equations (6) - (8) follow directly.

An illustration of these ideas is given by the determination of the enthalpy of binding oxygen to hemoglobin (ref. 14). We have seen that for this case $Q=[M] \sum \beta_{i} x^{i}$. Using equation (7) in conjunction with the van't Hoff equation, $\partial \beta_{i} / \partial(1 / T)=\beta_{i} \Delta H_{i}$ we find for a non-dissociating macromolecule that

$$
H_{m}-H_{m}^{0}=\frac{\sum \beta_{i} x^{i} \Delta H_{i}}{t \sum \beta_{i} x^{i}}
$$

In Fig 3 are shown the results of calorimetric experiments involving the titration of hemoglobin with oxygen using a thin layer sample calorimeter (ref. 15, 16).. The enthalpic data follow the general path already seen for the oxygen binding curve. The data in Fig 3 were obtained using a thin sample layer gas reaction calorimeter depicted in Figure 4. 

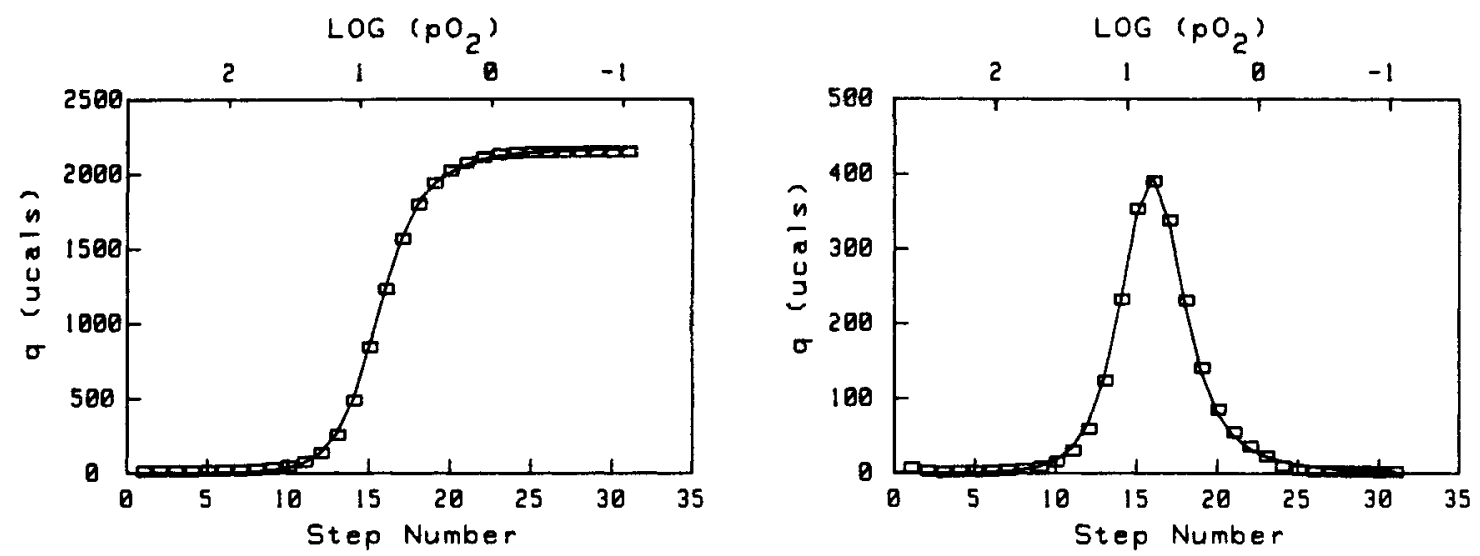

Fig. 3 (Left) Total enthalpic binding curve of oxygen to hemoglobin $A_{0}$ at $20^{\circ} \mathrm{C}$. Cumulative heat from logarithmic step dilutions. Data points ( $\square$ ), solid line theoretical best fit.. (Right) Enthalpy change for logarithmic step change in oxygen partial pressure Sample of 204 nmoles heme in $0.1 \mathrm{M}$ potassium phosphate buffer, $\mathrm{pH} 7.5$, heme concentration $12 \mathrm{mM}$. Best fit values: $\beta_{1}=0.10$ torr $^{-1}, \beta_{2}=0.016$ torr $^{-2}, \beta_{3}=2.4 \times 10^{-5}$ torr $^{-3}, \beta_{4}=5.3 \times 10^{-4}$ torr $^{-4}, \Delta H_{i}=i \Delta h$, with $\Delta \mathrm{h}=-11700 \mathrm{cal} \mathrm{mol}^{-1}$ (ref. 15). Note similarity with top panels of Fig. 1 .

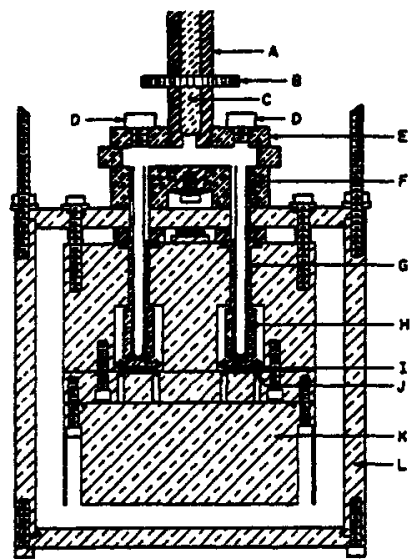

Fig. 4 Thin layer gas calorimeter. Sample is contained in the form of $1 \mathrm{~cm}^{2}$ piece of filter paper within an insertable NMR tube (F) of $4 \mathrm{~mm}$ diameter. The gas dilution valve (E) with entry ports (D), oxygen electrode (C), holder (A) and gear drive (B) at the top of the calorimeter container produces logarithmically spaced oxygen partial pressures, similar to the description in Fig. 2. Calorimeter copper cell $(\mathrm{H})$ held to thermoelectric units (I) by Nylon screws (J). Copper thermal block (K) located within stainless steel submarine (L) (taken from ref. 14).

\section{Stoichiometric representation of reactions with two ligands, $X$ and $Y$}

An important situation occurs when two different ligands bind to a macromolecule which serves to mediate the binding properties of each ligand. This type of linkage phenomena is important in control and regulatory processes in biochemical systems.

The underlying reaction processes can be represented by the formation reactions of various stoichiometric ligated species between $M$ and ligands $X$ and $Y$. The reaction species array is given as

$$
\begin{aligned}
& \mathrm{M} \rightarrow \mathrm{MX} \quad \rightarrow \mathrm{MX}_{2} \quad \rightarrow \ldots \rightarrow \mathrm{MX}_{\mathrm{t}} \\
& \stackrel{\downarrow}{\mathrm{M}} \rightarrow \stackrel{\downarrow}{\mathrm{M}} \mathrm{XY} \rightarrow \stackrel{\downarrow}{\mathrm{M}} \mathrm{X}_{2} \mathrm{Y} \rightarrow \ldots \rightarrow \stackrel{\downarrow}{\mathrm{M}} \mathrm{X}_{\mathrm{t}} \mathrm{Y} \\
& \stackrel{\downarrow}{\mathrm{M}} \mathrm{Y}_{2} \rightarrow \stackrel{\downarrow}{\mathrm{M}} \mathrm{XY}_{2} \rightarrow \stackrel{\downarrow}{\mathrm{M}} \mathrm{X}_{2} \mathrm{Y}_{2} \rightarrow \ldots \rightarrow \stackrel{\downarrow}{\downarrow} \mathrm{XX}_{1} \mathrm{Y}_{2}
\end{aligned}
$$

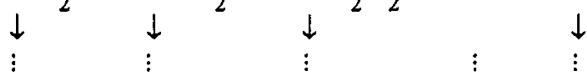

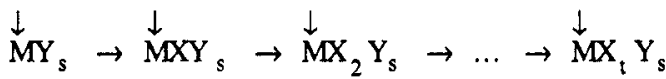


where there are $\mathrm{t}$ binding sites for ligand $\mathrm{X}$ and $\mathrm{s}$ binding sites for ligand $\mathrm{Y}$. The general reaction which characterizes the formation of particular species $M X_{i} Y_{j}$ is

$$
M+i X+j Y \rightarrow M X_{i} Y_{j}
$$

with an overall equilibrium constant $\beta_{\mathrm{ij}}$ which determines the concentration of $\mathrm{MX}_{\mathrm{i}} \mathrm{Y}_{\mathrm{j}}$ :

$$
\left[\mathrm{MX}_{\mathrm{i}} \mathrm{Y}_{\mathrm{j}}\right]=[\mathrm{M}] \beta_{\mathrm{ij}} \mathrm{x}^{\mathrm{i}} \mathrm{y}^{\mathrm{j}}
$$

where $x$ and $y$ are the $X$ and $Y$ ligand activities and $[M]$ is the unliganded macromolecule. The binding partition function for this situation is given as

$$
Q=\Sigma[M] \beta_{i j} x^{i} y^{j}
$$

where the summation is taken over all values of $i$ and $j$. The binding polynomial, $P$, introduced by Wyman (ref. 1, 10) for a non-dissociating macromolecule, is simply the binding partition function normalized to the concentration of the unligated species, $i$. e. $P=\Sigma \beta_{i j} x^{j} y^{j}$ and $\beta_{o o}=1$. The molar subunit concentration $m$ equals $r Q$. The amounts of $X$ and $Y$ ligand bound, $X_{m}$ and $Y_{m}$, are given by application of equation (3) for $X$ and its analog for $Y$ :

$$
\begin{aligned}
& X_{m}=\frac{1}{t} \frac{\partial \ln Q}{\partial \ln x}=\frac{1}{t} \frac{\sum i \beta_{i j} x^{i} y^{j}}{\sum \beta_{i j} x^{i} y^{j}}=-\frac{\partial \mu_{M}}{\partial \mu_{x}} \\
& Y_{m}=\frac{1}{s} \frac{\partial \ln Q}{\partial \ln y}=\frac{1}{s} \frac{\sum j \beta_{i j} x^{i} y^{j}}{\sum \beta_{i j} x^{i} y^{j}}=-\frac{\partial \mu_{M}}{\partial \mu_{y}}
\end{aligned}
$$

These equations reveal how the binding of either $\mathrm{X}$ or $\mathrm{Y}$ are linked to each others activities. The linkage coefficient, designated by $\Gamma$, is expressed by the derivative of $X_{m}$ with respect to $\mu_{\mathrm{y}}$ which is equivalent to the derivative of $Y_{m}$ with respect to $\mu_{\mathrm{x}}$ (ref. 17):

$$
\Gamma=\frac{\partial X_{m}}{\partial \mu_{y}}=\frac{\partial Y_{m}}{\partial \mu_{x}}=-\frac{\partial^{2} \mu_{M}}{\partial \mu_{x} \partial \mu_{y}}=-\frac{\partial^{2} \mu_{M}}{\partial \mu_{y} \partial \mu_{x}}
$$

The operational linkage coefficient $\Gamma \cdot R T$ is given by partial derivatives with respect to $\ln x$ and $\ln y$ :

$$
\Gamma \cdot R T=\frac{\partial X_{m}}{\partial \ln y}=\frac{\partial Y_{m}}{\partial \ln x}
$$

If the binding of $X$ and $Y$ are completely independent of each other then $\Gamma$ is zero. If one ligand assists the binding of the other then the linkage is positive and if it opposes the binding of the other then the linkage is negative.

Homotropic cooperativity is the effect a ligand of given species has on additional binding of the same ligand and is reflected in the slope of a binding curve. In analogy to the heat capacity, one may define the binding capacity (ref. 9) of $X$ at a given chemical potential of $Y,{ }^{S_{\mu}}$, by

$$
\mathrm{B}_{\mu_{\mathrm{Y}}}=\frac{\partial \mathrm{X}_{\mathrm{m}}}{\partial \mu_{\mathrm{x}}}=\frac{1}{\mathrm{RT}} \frac{\partial \mathrm{X}_{\mathrm{m}}}{\partial \ln \mathrm{x}}
$$

Similarly the binding capacity of $Y$ at fixed chemical potential of $X,{ }^{E_{\mu}}{ }_{X}$, is given by

$$
E_{\mu_{X}}=\frac{\partial Y_{m}}{\partial \mu_{y}}=\frac{1}{R T} \frac{\partial Y_{m}}{\partial \ln y}
$$



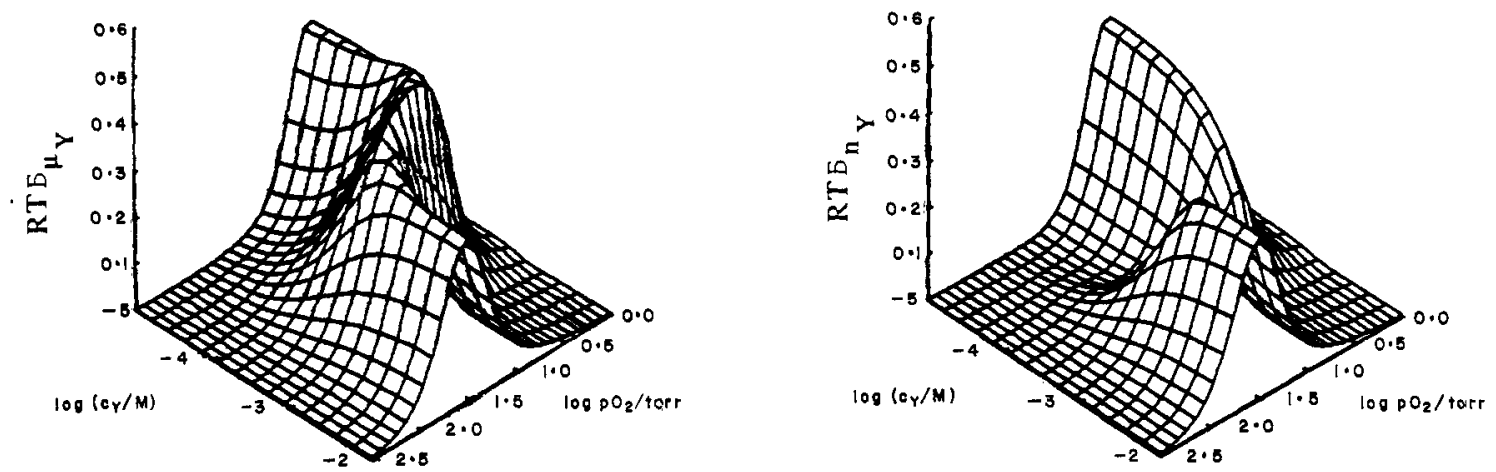

Fig. 5. Oxygen binding to human hemoglobin in the presence of inositol hexaphosphate (IHP). (Left) Oxygen binding capacity per mole of heme, RT ${ }_{\mu_{Y}}$ as a function of logarithmic oxygen pressure and IHP concentration ( $C_{Y}$ ) under various fixed chemical potential of IHP. (Right) Oxygen binding capacity per mole of heme, RTE $n_{Y}$ as a function of oxygen and IHP concentration under fixed amount of IHP. Hemoglobin concentration used in these studies was $4 \mathrm{mM}$ heme. (ref. 18)

One may also define a binding capacity coefficient in terms of fixed amount of the alternate ligand, much as one does the heat capacity at constant volume. In all linkage situations the rules of thermodynamics require that the binding capacity coefficient at fixed ligand chemical potential, $B_{H_{Y}}$, is greater than that at fixed amount of ligand, ${ }^{B_{\mathrm{Y}}}$ (ref. 9)

The binding properties of oxygen to hemoglobin in the presence of varying amounts of organic phosphates, diphosphoglycerate (DPG) and inositol hexaphosphate (IHP) have been studied at high hemoglobin concentration in detail in our laboratory (ref 18). A single organic phosphate molecule may be bound along with the four oxygen molecules.

The reactions are important physiologically to provide regulation of oxygen binding at high altitudes. Some of the results using IHP, which shows the more dramatic effect, are shown in Figure 5, in the form of oxygen binding capacities under fixed chemical potential of IHP or under fixed amount of IHP. We see that the constant chemical potential binding capacity is everywhere greater than the constant amount binding capacity, i.e., the cooperativity is largest in a well buffered system.

\section{Identical linkage}

$$
\begin{aligned}
& \mathrm{MY}_{\mathrm{s}} \rightarrow \mathrm{MXY}_{\mathrm{s}} \rightarrow \mathrm{MX}_{2} \mathrm{Y}_{\mathrm{s}} \rightarrow \ldots \rightarrow \mathrm{MX}_{\mathrm{t}} \mathrm{Y}_{\mathrm{s}} \\
& \mathrm{M} \rightarrow \mathrm{MX} \quad \rightarrow \ldots \quad \rightarrow \mathrm{MX}_{\mathrm{t}-1} \rightarrow \mathrm{MX}_{\mathrm{t}} \\
& \begin{array}{l}
\downarrow \\
\mathrm{MY}
\end{array} \underset{\substack{\mathrm{MXY} \\
\downarrow}}{\downarrow} \rightarrow \ldots \quad \rightarrow \stackrel{\downarrow}{\mathrm{MX}_{\mathrm{t}-1} \mathrm{Y}} \\
& \text { : } \quad \vdots \quad \therefore \\
& \begin{array}{l}
\downarrow \\
\mathrm{MY}_{\mathrm{t}-1} \\
\mathrm{MY}_{\mathrm{t}}
\end{array} \rightarrow \stackrel{\downarrow}{\mathrm{MXY}}{ }_{\mathrm{t}-1}
\end{aligned}
$$

A simple but important situation occurs when the binding of $X$ or $Y$ occurs to the same site. This is called identical linkage (ref. 19). The general array of reaction species denoted by equation (12) must then be square $(t=s)$ and the maximum number of both ligands bound is $t$. The array is then triangular in form shown in (22). 

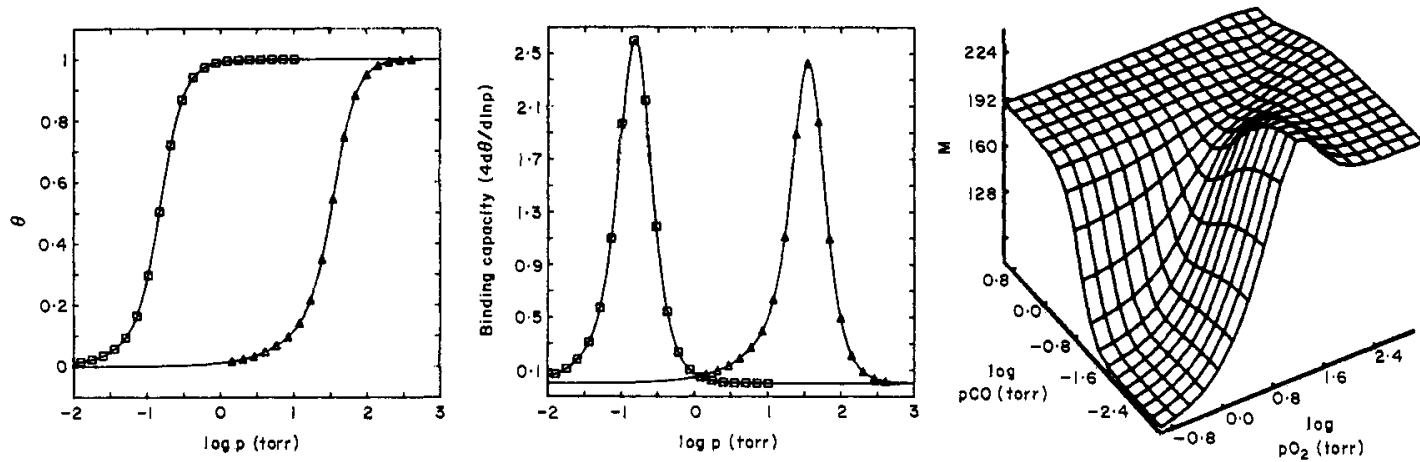

Fig. 6. Oxygen and carbon monoxide measurements to human hemoglobin as a function of logarithm partial pressures (ref. 21). (Left) Binding curves $\left(\Theta=X_{m}\right.$ or $Y_{m}$ for oxygen $(X)(\Delta)$ and carbon monoxide (Y) (a)as a function of respective partial pressures. (Middle) Binding capacity measurements along with best fit representation. (Right) Haldane coefficient, $m=\left(X_{m} / Y_{m}\right)(y / x)$, shown as function of logarithmic values of oxygen $(x)$ and carbon monoxide ( $y$ ) activities. Note that the cooperativity of the carbon monoxide is higher than that of oxygen and that the Haldane coefficient depends strongly upon the ligand activities (or degrees of saturation).

An important application of this scheme is where oxygen and carbon monoxide bind to the same heme site in hemoglobin. Detailed studies of these reactions have been made possible by use of the thin layer technique (ref. 11). The binding capacity curves, which are actually obtained directly by this method, are shown in Fig. 6 along with the then generated full binding curves for the two pure ligands. The differences in the binding of the oxygen and carbon monoxide to hemoglobin are brought out more forcefully by Haldane's coefficient $\left[\mathrm{m}=\left(\mathrm{X}_{\mathrm{m}} / \mathrm{Y}_{\mathrm{m}}\right)(\mathrm{y} / \mathrm{x})\right]$ (ref. 20). This coefficient reflects the competitive binding properties of the two ligands, and is also plotted in Fig. 6 as a function of logarithmic values of oxygen and carbon monoxide partial pressure. The construction of this Haldane coefficient plot utilized results from additional experiments in which oxygen binding was studied in the presence of different partial pressures of carbon monoxide. One sees immediately that the value of $m$ depends on ligand conditions, and thus one concludes that the hemoglobin macromolecule has a very different response to eacb of these gaseous binding substances.

In the case of hemoglobin with its four heme binding sites, it is possible to analyze the experimental results in terms of the operational stoichiometric binding constants for these particular situations of identical linkage. When there are more than four reaction sites then this type of analysis is usually not practical, and one must turn to models incorporating additional ideas. One of the most useful ideas in this regard is that of conformational equilibria between different allosteric forms - a concept first suggested by Wyman (ref. 1).

\section{Allosteric reaction processes}

The concept that macromolecules can exist in well-defined different thermodynamic states, each with particular physical and chemical properties, was introduced by Wyman and Allen (ref. 22) to explain the Bohr effect, the effect of $\mathrm{pH}$ on the oxygen binding properties of hemoglobin. The idea, described usually as the allosteric concept, has broad application. Here we shall only outline its use in describing homotropic cooperativity.

Equilibria between various conformational or allosteric states of a macromolecule which binds ligand $X$ is depicted by the reaction scheme in equation(23). Here for the sake of emphasis we have noted the allosteric form by $A_{k}$ which can attach various numbers of $\mathrm{X}$ ligand. By our general subscript convention this is equivalent to a species $M_{k} X_{i}$. The equilibrium reactions for the unliganded allosteric forms, the top row, are $A_{0} \rightarrow A_{k}$ with an equilibrium constant given by $L_{k}$. The $X$ ligand binding reaction to $A_{k}$ is $A_{k}+i X \rightarrow$ $\mathrm{A}_{k} \mathrm{X}_{\mathrm{i}}$ with an equilibrium constant given by $\beta_{\mathrm{ki}}$. The general reaction process, starting 
from the unliganded form in the top left corner, is then $A_{o}+i X \rightarrow A_{k} X_{i}$ and its overall equilibrium constant is $L_{k} \beta_{k j}$.

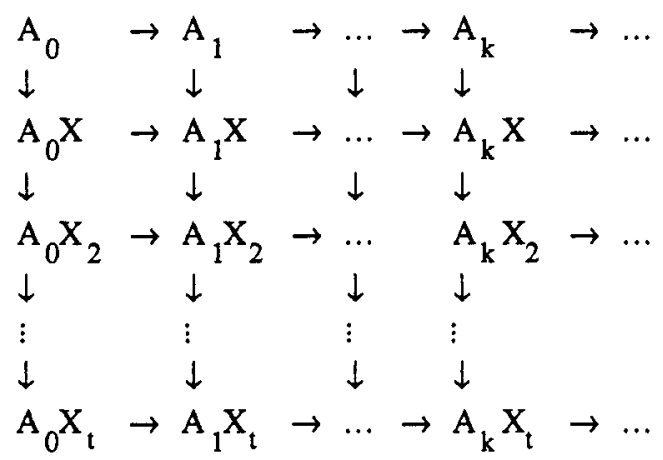

The concentration of any given species in the array may then be calculated from the left corner reference state by $\left[A_{k} X_{i}\right]=\left[A_{0}\right] L_{k} \beta_{k i} x^{i}$. The form of the allosteric partition

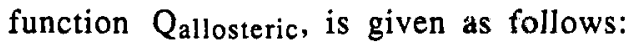

$$
\mathrm{Q}_{\text {allosteric }}=\left[\mathrm{A}_{\mathrm{o}}\right] \sum_{\mathrm{k}} \mathrm{L}_{\mathrm{ki}} \mathrm{x}^{\mathrm{i}}
$$

where the summation is taken over all allosteric forms (k) and $X$ ligand stoichiometries (i). The extension to a second ligand $\mathrm{Y}$ is accomplished by viewing the reaction scheme as a three dimensional lattice of species including the various $\mathrm{Y}$ ligated allosteric forms.

Monod, Wyman, and Changeux (ref. 23) described the situation for only two allosteric forms designated by $R$ and $T$ in which the $X$ ligand binding constants were choosen to describe reactions with $r$ independent identical sites with affinities depending only on the allosteric form, $\kappa_{R}$ or $\kappa_{T}$. This set of simple conditions gives $Q_{M W C}$ :

$$
\mathrm{Q}_{\mathrm{MWC}}=\left[\mathrm{A}_{\mathrm{O}}\right]\left(\left(1+\kappa_{\mathrm{R}} \mathrm{x}\right)^{\mathrm{T}}+\mathrm{L}\left(1+\kappa_{\mathrm{T}} \mathrm{x}\right)^{\mathrm{T}}\right)
$$

The amount of ligand bound $X_{m}$, the Hill coefficient, or the binding capacity may be readily calculated from this expression using equations (3), (4), or (5). The inclusion of a second identically linked ligand $Y$, which also binds independently with binding constants, $\lambda_{\mathrm{R}}$ and $\lambda_{\mathrm{T}}$, that depend only on the allosteric form, requires only the simple following modification of equation (25):

$$
\mathrm{Q}_{\mathrm{MWC}}=\left[\mathrm{A}_{\mathrm{O}}\right]\left(\left(1+\kappa_{\mathrm{R}} \mathrm{x}+\lambda_{\mathrm{R}} \mathrm{y}\right)^{\mathrm{T}}+\mathrm{L}\left(1+\mathrm{\kappa}_{\mathrm{T}} \mathrm{x}+\lambda_{\mathrm{T}} \mathrm{y}\right)^{\mathrm{T}}\right)
$$

The amounts of ligand $X$ and $Y$ can be found by applying equations (16) and (17), the linkage between these ligands is expressed by the heterotropic derivatives given by equation (18), and the binding capacities are determined by the homotropic derivatives of the form of equations (20) and (21).

The most extensive application of the MWC allosteric model has been directed to hemoglobin. But it fills an important need in providing a fitting model for much more complex situations found for example with large hemocyanin multi-subunit molecules.

In the case of the hemocyanin found in Octupus dofleini there are 70 oxygen binding sites $(t=70)$, which come from the assembly of 10 polypeptide chains, each with 7 binuclear copper sites where oxygen binding occurs (ref. 24). A recent study (ref. 25) of oxygen and carbon monoxide binding to this complex molecule shows that the MWC model describes the binding processes, provided one takes the cooperative allosteric unit with a size of seven units. This essentially means that the partition function for the complete decamer is made up of independent MWC units and thus has the partition function $Q$ given by

$$
Q=\left\{Q_{M W C}\right\}^{10}=\left[A_{0}\right]\left\{\left(\left(1+\kappa_{R} x+\lambda_{R} y\right)^{7}+L\left(1+\kappa_{T} x+\lambda_{T} y\right)^{7}\right)\right\}^{10}
$$

where in this last equation $\left[A_{0}\right]$ now represents the concentration of the unligated 70 mer species. Thin layer binding studies using $\mathrm{O}_{2}$ and $\mathrm{CO}$ have shown that this partition function describes the functional properties of Octupus dofleini. The results enable 
representation of these functional properties in three dimensional graphs shown as the binding potential per mole of subunit $\left[\pi=-t \mu_{M}=R T \ln Q\right]$, and the binding curves of $X_{m}$ and $Y_{m}$ as a function of partial pressures as shown in Fig. 7.

The complete functional representation is contained in the binding potential surface, whose first derivative slopes determine the amounts of the two ligands bound, and whose second derivatives give the binding capacities and linkage coefficient. The cooperativity is revealed more visually by the Hill slope, directly obtained from the binding capacities. As seen in Fig. 8 the relative large value of the oxygen binding Hill slope shows the cooperative nature of hemocyanin for this ligand.in the absence of carbon monoxide. The smaller Hill slope of carbon monoxide reveals its lack of cooperativity, although in the presence of sufficient oxygen carbon monoxide binds with increasing cooperativity. The negative nature of the linkage coefficient demonstrates the fundamental competitive nature of the two ligands and its magnitude increases with increasing degree of saturation.

What we see here with Octupus dofleini hemocyanin is found to various degrees in other studies of hemocyanins from lobster (ref. 26) and tarantula (ref. 27) However the allosteric mechanism involved in those cases appears to involve long range interactions which can be described (ref. 28) by nested allosteric conformational changes originally proposed by Wyman (ref. 29).

\section{Aggregation reaction processes}

A natural progression from our considerations of equilibria between ligand mediated allosteric forms is to recognize an extreme form of conformational change occurs upon aggregation of subunits of a multi-subunit macromolecule. The binding properties of the aggregate are in general different than the isolated subunits. We can outline the elements of these systems by considering the following reaction scheme: first, the formation of unligated aggregate forms, designated by $\mathrm{M}_{\mathrm{h}}$, is described by the reaction process $\quad M \rightarrow(1 / h) M_{h}$, with equilibrium constant $\Lambda_{h}$; second, the binding of $i X$ ligands is described by $M+i X \rightarrow(1 / h) M_{h} X_{i}$ with an equilibrium constant denoted by $\beta_{h i}$.

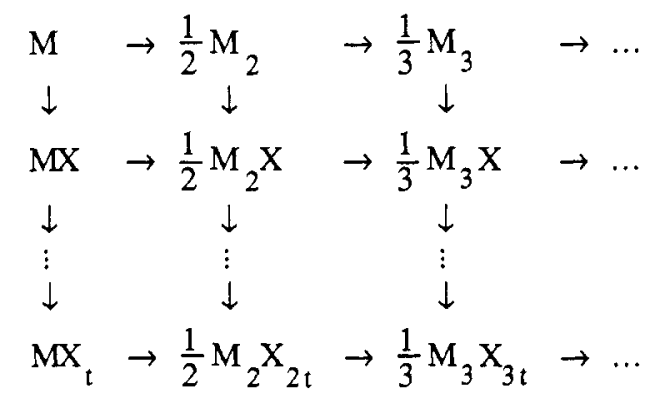

The concentration of a given aggregate is $\left[\mathrm{M}_{h} \mathrm{X}_{\mathrm{i}}\right]=[\mathrm{M}]^{\mathrm{h}}\left(\Lambda_{\mathrm{h}}\right)^{\mathrm{h}} \beta_{\mathrm{hi}} \mathrm{X}^{\mathrm{i}}$ and the partition function Qaggregate is

$$
Q_{\text {aggregate }}=\sum\left[\mathrm{M}^{\mathrm{h}}\left(\Lambda_{\mathrm{h}}\right)^{\mathrm{h}} \beta_{\mathrm{hi}} \mathrm{x}^{\mathrm{i}}\right.
$$

where the summation is over all species in the array (28). A significant complexity is present here not encountered in the previous situations we have described - namely the relative species concentrations depends upon the total concentration. In other words the solvent ligand affects the equilibrium properties of the aggregated system. At very low subunit concentrations one is dealing essentially with the pure subunit binding properties, and at very high subunit concentration there will be an emphasis on the properties of the largest aggregate that can form. One of the most well characterized situations of this type is the aggregation reaction of $\alpha \beta$ dimer subunits of hemoglobin to form the $\alpha_{2} \beta_{2}$ tetramer. The oxygen linked aggregation properties of hemoglobin have been studied in detail by Ackers and coworkers (ref. 30). An even simpler case is that of the dimeric cytochrome c' from Chromatium vinosum which binds carbon monoxide to its heme containing subunits. In the presence of carbon monoxide the association equilibria of the scheme (28) is shifted strongly to the monomer form. The carbon monoxide binding is both cooperative and sensitive to protein concentration (ref. 31) 

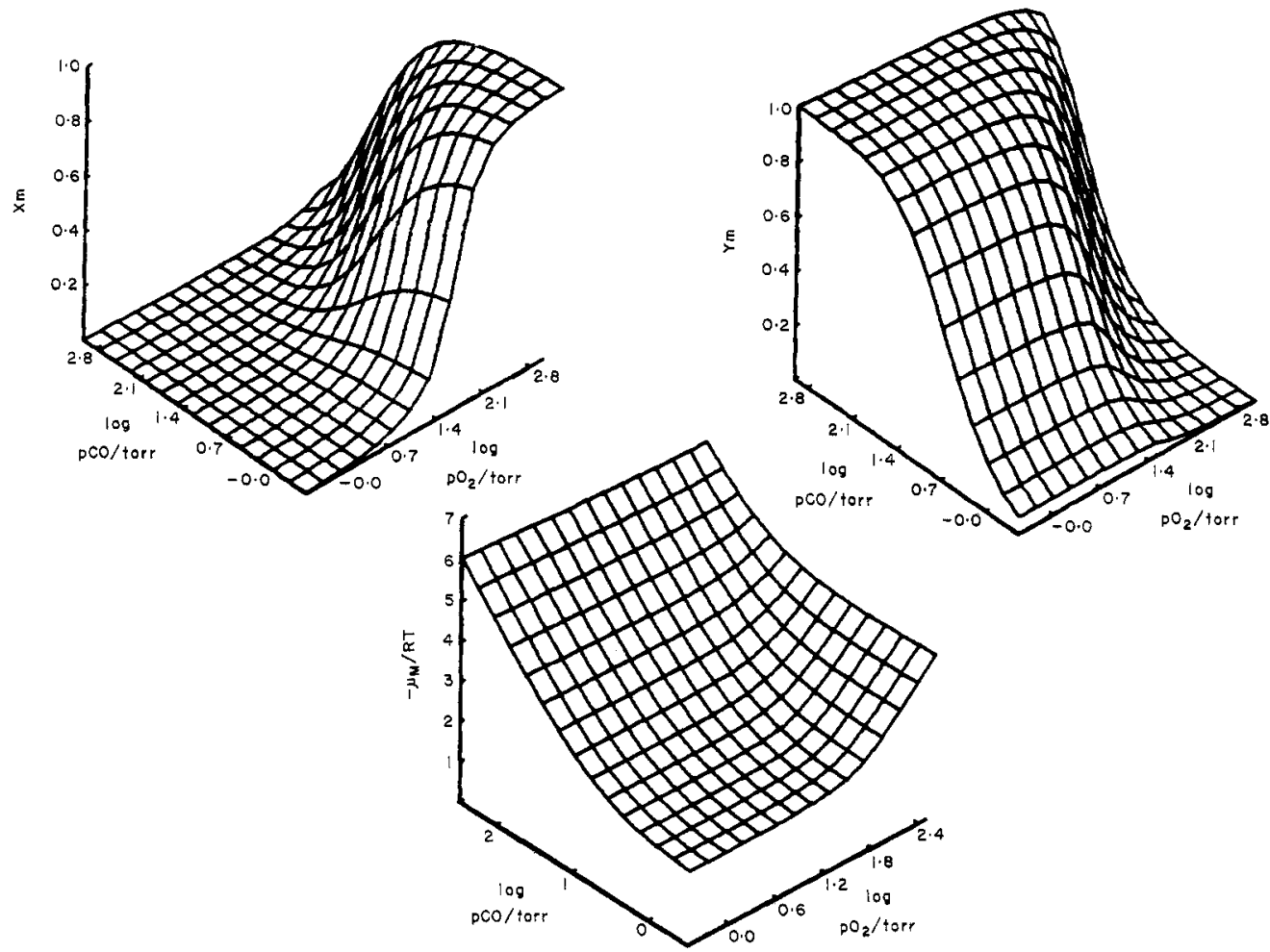

Fig. 7. Oxygen and carbon monoxide functional properties of Octupus dofleini hemocyanin (ref. 25). (Bottom) Subunit chemical potential, - $\mu_{M}$, versus logarithmic values of oxygen $(X)$ and carbon monoxide $(Y)$, partial pressures in Torr. (Top) Binding curves of oxygen (left) and carbon monoxide (right) per reaction site, as a function of logarithmic partial pressures.

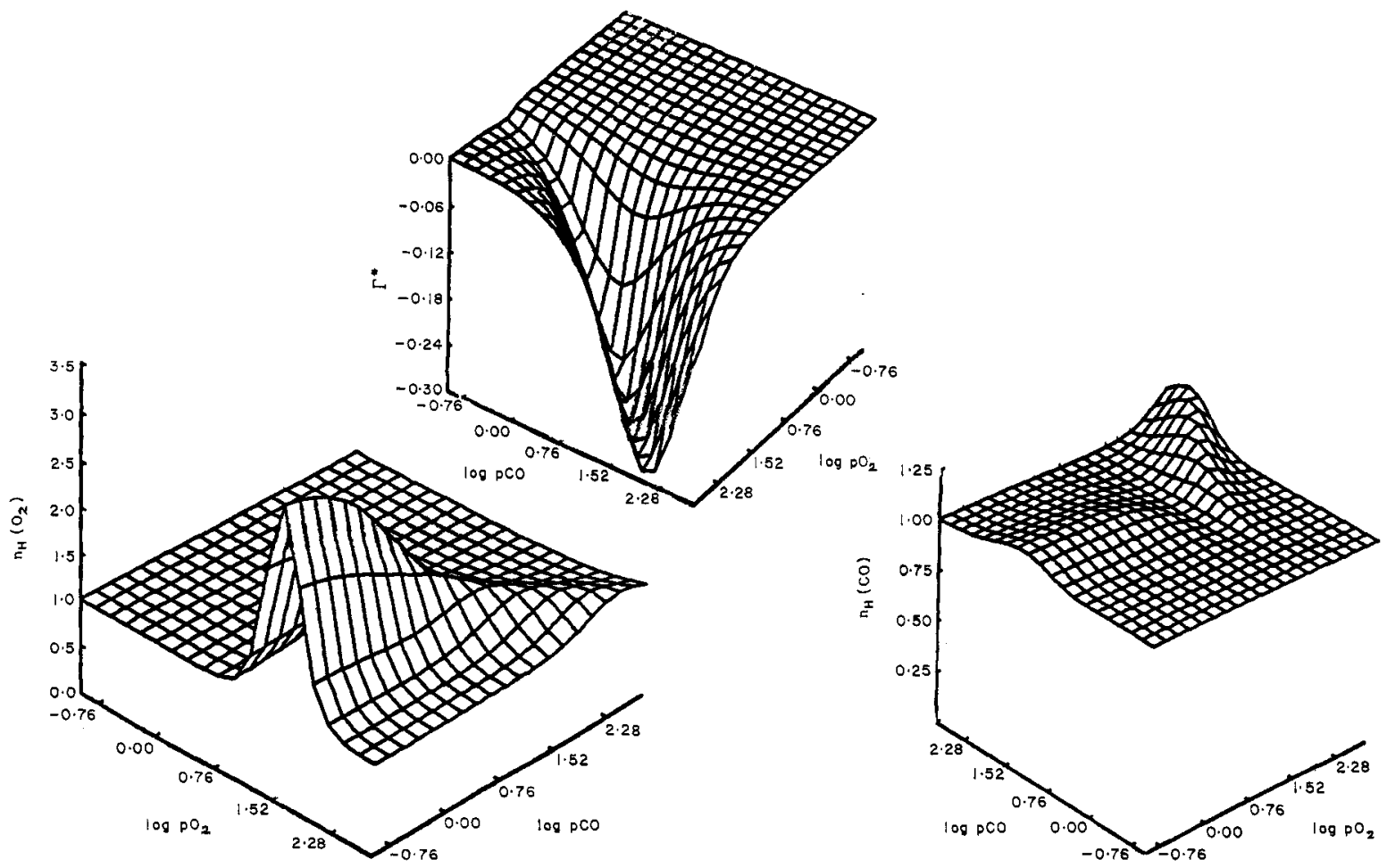

Fig. 8. Oxygen and carbon monoxide functional properties of Qctupus dofleini hemocyanin (ref. 25). (Top) Site normalized linkage coefficient $\Gamma^{*}=\Gamma R T$ versus logarithmic values of oxygen $(\mathrm{X})$ and carbon monoxide (Y), partial pressures in Torr.. (Bottom) Hill slope coefficients $\mathrm{n}_{\mathrm{H}}$ for oxygen (left) and carbon monoxide (right) per reaction site, as a function of logarithmic partial pressures. 


\section{CONCLUSIONS}

We have seen how the macromolecular binding partition function (Q) serves as a powerful yet simple vehicle for formulating complex equilibrium reactions with macromolecules in either non-aggregating or aggregating conditions. Amounts of bound chemical ligand, enthalpy, and volume changes are calculated from appropriate derivatives of $Q$. $Q$ is directly related to the macromolecular chemical potential and while first derivatives yield amounts of extensive properties bound per mole of subunit, second derivatives describe the more interesting properties of cooperativity and linkage. Application of these concepts requires the availability of high precision binding measurements, taken under a variety of conditions. So far the best examples are found with respiratory protein systems, such as hemoglobin and hemocyanin, where one can employ gaseous ligands to unparalleled degrees of precision. Data obtained on these systems enable tests of structurally motivated allosteric models, and thus a critical basis for evaluating the suitability of such models.

\section{Acknowledgements}

This work was supported by NIH Grant HL 22325. I want to acknowledge the strong impact of Jeffries Wyman over the past decade on our work together. Without the assistance of a number of doctoral students, Chuck Robert, Mike Doyle, Pat Connelly, Gary Bishop, and Brough Richey, and postdoctoral fellows, Antonio Parody-Morreale, Heinz Decker, and Enrico Di Cera, this work could not have been done. A special thanks goes to Chuck Robert for his extensive help in preparing the three-dimensional representations of binding and linkage phenomena.

\section{REFERENCES}

1. J. Wyman, Adv, Protein Chem. 4, 407-531 (1948).

2. J. Herzfeld and H. E. Stanley, J. Mol. Biol. 82, 231-265 (1974)

3. J. A. Schellman, Biopolymers 14, 999-1018 (1975).

4. S. J. Gill, Biochemical Thermodynamics Vol 1, Edited by M. N. Jones, Elsevier, Amsterdam, 224-255 (1979).

5. J. Wyman, J.Mol. Biol. 11, 631-44 (1965).

6. T. L. Hill, Introduction to Statistical Thermodynamics, Addison-Wesley, Reading (1960)

7. S. J. Gill, P. R. Connelly, E. Di Cera, and C. H. Robert, Biophys, Chem. 30, 133-141

(1988).

8. S. J. Gill, E. Di Cera, M. L. Doyle, G. A. Bishop, and C. H. Robert, Biochemistry 26, 3995 4002 (1987).

9. E Di Cera, S. J. Gill, and J. Wyman, Proc. Natl. Acad. Sci. USA 85, $449-452$ (1988)

10. J. Wyman, Adv. Protein. Chem, 19, 223-286 (1964).

11. D. Dolman and S. J. Gill, Anal. Biochem. 87, 127-134 (1978).

12. S. J. Gill, B. Richey, G. Bishop, and J. Wyman, Biophys. Chem., 21, 1-14 (1985).

13. S. J. Gill, C. H. Robert, and J. Wyman, Biochemical Thermodynamics (second edition) edited byM. N. Jones, chapter 4, Elsevier, Amsterdam, (1988).

14. M. L. McGlashan, Chemical Thermodynamics, Academic Press, London, p. 77 (1979).

15. G. A. Bishop, Ph. D. Thesis, Univ. of CO. (1986)

16. G. A. Bishop, A. Parody-Morreale, C. H. Robert, and S. J. Gill, Rev. Sci. Instrum. 58, 632.638 (1987)

17. .E. Di Cera, S. J. Gill, and J. Wyman, Proc. Natl. Acad. Sci. USA 85, 5077-5081 (1988)

18. C. H. Robert, L. Fall, and S. J. Gill, Biochemistry 27, (in press) (1988)

19. J. Wyman, Quart. Rev. Biophys, 1, 35-80 (1968).

20. J. S. Haldane and J. L. Smith, J. Physiol. (London) 22, $231-258$ (1897)

21. E. Di Cera, M. L. Doyle, P. R. Connelly, and S. J. Gill, Biochemistry 26, 6494-6502 (1987).

22. J. Wyman and D. W. Allen, L.Polymer Sci. 7, 499-518 (1950)

23. J. Monod, J. Wyman, and J. P. Changeux, J. Mol. Biol. 12, 88-112 (1965)

24. K. Miller, Biochemistry 24, 4582-4586 (1985)

25. P. R. Connelly, Ph. D. Thesis, University of Colorado, 1988

26. B. Richey, H. Decker, and S. J. Gill, Biochemistry 24, 109-117 (1985)

27. H. Decker, C. H. Robert, and S. J. Gill, in Invertebrate Oxygen Carriers Symposium Proceedings, ed. B. Linzen (Springer, Berlin), 383-388 (1986)

28. C. H. Robert, H. Decker, B. Richey, S. J. Gill, and J. Wyman, Proc. Natl. Acad. Sci. USA 84. $1891-1895$ (1987)

29 J. Wyman, Quart. Rev. Biophys. 17, 543-488 (1984)

30. F. C. Ackers, M. L. Johnson, and G. K. Ackers, Biochemistry 15, 5350-5362 (1976)

31. M. L. Doyle, S. J.Gill, and M. A. Cusanovitch, Biochemistry 25, 2509-2516 (1986). 